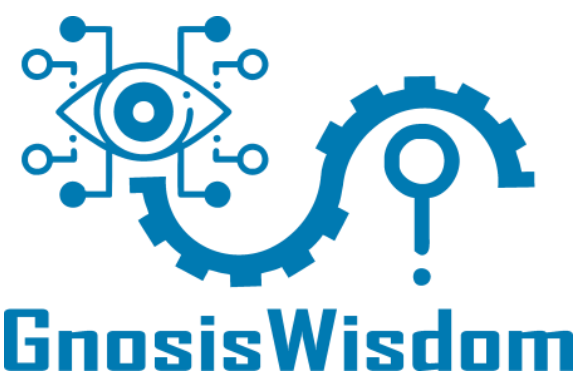

\title{
ACCIÓN DEL AGUA Y LA TEMPERATURA AMBIENTAL EXTREMA SOBRE PAVIMENTO FLEXIBLE
}

\section{ACTION OF WATER AND EXTREME AMBIENT TEMPERATURE ON FLEXIBLE PAVEMENTS}

\begin{abstract}
Autores
Alfredo, Rojas Carrizales

Universidad Nacional de Huancavelica

Yovana, Torres Gonzales

Universidad Nacional de Huancavelica

Miguel, Parejas Garavito

Universidad Nacional de Huancavelica

Rene, Hinojosa Benavides

Universidad Nacional Autónoma de Huanta
\end{abstract}

\section{Resumen}

Para el diseño de pavimentos flexibles se han incluido variables climáticas, pues se ha demostrado que es un factor que incide en el comportamiento estructural de las carreteras; siendo necesario incluir el efecto climático en el diseño de estructuras de pavimentos; en este sentido, el objetivo de esta investigación bibliográfica es mostrar que el nafteno, principal componente del asfalto, sufre los efectos climáticos del agua y la temperatura ambiental, al mismo tiempo, tiene una relación directa con el deterioro de la superficie flexible; sin embargo, existen métodos de prueba para el envejecimiento del asfalto, que intentan reproducir el efecto del agua y la temperatura ambiental sobre la mezcla de asfalto, con el fin de acelerar el proceso de envejecimiento del aglomerante. La inyección de oxígeno sobre el asfalto en caliente, genera mayor cantidad de asfaltenos en detrimento de los maltenos, provocando mayor resistencia ante temperaturas elevadas, variando las iniciales condiciones reológicas del asfalto. Las pruebas para el proceso de envejecimiento del asfalto por oxidación, permiten conocer la modificación de las propiedades físicas del asfalto, con el fin de mejorar su resistencia y adherencia en condiciones climáticas extremas, en el uso de pavimentos flexibles.

Palabras clave: Agua, temperatura ambiental, reología, asfalto. 


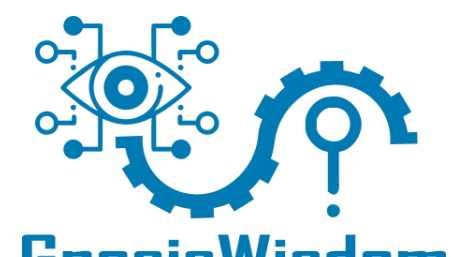

\section{REVISTA DE INVESTIGACIÓN E INNOVACIÓN}

CIENTÍFICA Y TECNOLÓGICA

Volumen 1, Número 2, Mayo-Agosto 2021

Fecha de publicación: 2021-08-18 Fecha de aceptación: 2021-08-02

\section{GnosisWisdam}

\section{Abstract}

For the design of flexible pavements, climatic variables have been included, because it has been shown that it is a factor that affects the structural performance of roads; being necessary to include the climatic effect in the design of pavement structures; in this sense, the objective of this bibliographic research is to show that naphthene, the main component of asphalt, suffers the climatic effects of water and environmental temperature, at the same time, it has a direct relationship with the deterioration of the flexible surface; however, there are test methods for aging asphalt, which attempt to reproduce the effect of water and ambient temperature on the asphalt mixture, in order to accelerate the aging process of the binder. Injecting oxygen onto a mass of asphalt, in hot produces a greater amount of asphaltenes to the detriment of maltenes, causing greater fragility, greater resistance to high temperatures and a variation of the initial rheological conditions of the asphalt. The tests for the aging process of the asphalt by oxidation, they allow to know modification of the physical properties of asphalt, in order to improve its resistance and adherence in extreme weather conditions, in the use of flexible pavements.

Keywords: Water, ambient temperature, rheology, asphalt.

\section{Introducción}

El diseño del pavimento que se basa en métodos empíricos, hoy en día es sustituido por métodos empírico-mecánicos, ya que esta metodología utiliza el estudio de tráfico, el clima y el diseño de la estructura para obtener las predicciones en el desempeño del pavimento, proporcionando el diseño adecuado en un determinado clima, donde prestará la serviciabilidad (Hirooka et al, 2019); por consiguiente, Mendoza y Marcos (2017) aseveran que existen evidencias en el diseño de estructuras viales y su construcción, en el que se ha incluido variables climáticas, y queda demostrado que el factor clima, también afecta el desempeño de las carreteras; no obstante, los desafíos de un clima cambiante, especialmente en regiones mayores a los 3000 m s.n.m. están forzando a tomarlos en cuenta en el diseño e incluso generar nuevos métodos para construir infraestructura de una vía resiliente, por la reacción del asfalto a los parámetros del agua y temperatura del clima; en tanto, Leyva et al. (2016) aseveran que es necesario e imprescindible considerar el efecto climático para diseño de estructuras de pavimento que están expuestas al ambiente, también se indica en cuanto a la regularidad de la superficie de rodadura, mediante el levantamiento del estado del comportamiento de superficial; por lo que en las estructuras de pavimento que están expuesta al efecto climático, es muy importante considerar el efecto climático.

La acción de oxidación en el proceso de envejecimiento del asfalto con la finalidad de estudiar sus propiedades de resistencia y adherencia; la composición química del asfalto se altera, esencialmente los asfaltenos y maltenos, en tanto, existen métodos para envejecer el asfalto, que tratan de reproducir los efectos que produce la presencia de agua y la temperatura ambiental sobre la mezcla asfáltica; al respecto menciona Ranura y Rommel (2019) un método como el Envejecimiento en Horno Rotacional de Película Delgada (RTFO), que permite simular el envejecimiento a corto plazo por volatilización, cuya muestra de asfalto se encuentra expuesta al aire y calor, lo cual se asemeja a lo que 


\section{GnasisWisdam}

ocurre durante el mezclado en caliente, transporte y colocación en la carpeta asfáltica; un segundo método es el envejecimiento en la Vasija a Presión (PAV) que simula el envejecimiento por oxidación que ocurre en ligantes asfálticos durante la vida de servicio del pavimento, donde el residuo proveniente de este ensayo puede ser usado para estimar las propiedades físicas o químicas de ligantes asfálticos después de cinco a diez años de envejecimiento en el campo.

Actualmente se desarrollan ensayos experimentales como el Ensayo Fénix (EF), para determinar la resistencia a la fisuración de la mezcla asfáltica, para conocer la energía que se disipa durante el proceso de fisuración, ya que una forma efectiva de evaluar el efecto que produce el agua sobre una mezcla semidensa, es a través de la medición de la resistencia a la fisuración de las mezclas bituminosas en el proceso de envejecimiento y resistencia que presenta a su fisuración; ciertamente, el ensayo Fénix, aun siendo experimental, permite valorar la resistencia a la fisuración de la mezcla (Bianchetto et al., 2020). El propósito del presente estudio es evidenciar que el nafteno, componente principal de asfalto, sufre los efectos climáticos del agua y la temperatura ambiental, así como también tienen una relación directa con el deterioro de la superficie del pavimento flexible.

\section{Material y método}

Se hizo una revisión bibliográfica en distintas bases de datos como repositorios de universidades, bases de datos como SciELO, Scopus, Dialnet plus y Web of Science, empleando descriptores como "agua" "clima extremo" "asfalto" "pavimentos", "temperatura ambiental", del mismo modo se desarrolló el estudio sistemático de artículos científicos publicados en revistas científicas de infraestructura vial, publicaciones técnicas sobre el clima y las carreteras por el Instituto Mexicano del Transporte, entre otros, desarrollando el estado de arte en sus fases tanto heurística como hermenéutica.

\section{Ensayo que induce el envejecimiento acelerado en pavimento flexible.}

Para estudiar las propiedades de resistencia y adherencia del asfalto, se le somete a un proceso de envejecimiento por oxidación, que altere la composición química de sus constituyentes, los asfaltenos y maltenos, en el que los maltenos actúan como la fase continua que dispersa a los asfaltenos. Durante la exposición del asfalto a altas temperaturas, sus propiedades físico-químicas cambian por un proceso de oxidación y envejecimiento del ligante, el cual incide de manera directa en el incremento de la rigidez y la disminución de la ductilidad (Guerrero, 2019). Entre las propiedades físicas de los asfaltos que se obtienen por destilación permiten ser dúctiles, maleables y reológicamente aptos para su utilización como materias primas para elaborar productos para el mercado vial. Al reaccionar el oxígeno del aire sobre una masa de asfalto en caliente aumenta la cantidad de asfaltenos en detrimento de los maltenos, consecuentemente provoca una mayor fragilidad, mayor resistencia a altas temperaturas y una variación de las condiciones reológicas iniciales, según Arellano y Cáceres (2018) para pavimentos que se encuentren a más de 3000 m s.n.m. es primordial colocar carpetas mayores a 3" en una sola capa a fin de hacerle frente al gradiente térmico que fluctúa entre $-15^{\circ} \mathrm{C}$ a $20^{\circ} \mathrm{C}$ y que la compactación se desarrolle mientras la mezcla 


\section{GnasisWisdam}

asfáltica en caliente se encuentre dentro de un rango de $120^{\circ} \mathrm{C}$ a $130^{\circ} \mathrm{C}$

Entre las metodologías que permiten realizar el envejecimiento del asfalto, son el horno de película delgada rotatoria, o soplar el asfalto para producir un material con aplicaciones de uso industrial, en todos ellos siempre se toma en cuenta el proceso de envejecer el ligante. Al respecto Vacca et al. (2012) afirman que la rigidez de la mezcla asfáltica original en los valores de módulo resiliente para temperaturas bajas $\mathrm{y}$ frecuencias de aplicación altas. Los módulos resilientes a $20^{\circ} \mathrm{C}$ y $30^{\circ} \mathrm{C}$ presentan incrementos de hasta el $21 \%$, y es más rígida la mezcla con asfalto envejecido.

Existe un probador automático de contenido de asfalto, desarrollado por la National Center for Asphalt Technology (NCAT), un equipo con las condiciones óptimas para implementar un ensayo de estas características, diseñado para obtener el contenido de asfalto de una mezcla por ignición, en donde las condiciones de operación pueden modificarse para que el horno solo envejezca el asfalto y no lo calcine; de tal manera que, se someten probetas disgregadas con $6 \%$ de asfalto y 1100 gramos de agregados pétreos con mezclas diseñadas para asfalto normal CA $60-80$ y asfalto modificado Cariphalte MD 60-80, a temperaturas constantes de $135{ }^{\circ} \mathrm{C} \quad$ y $\quad 165{ }^{\circ} \mathrm{C}$ respectivamente, en intervalos de tiempo de entre 4 a 6 h.

Actualmente los hornos de ignición usan transferencia de calor por convección, requiriéndose que la cámara de aire que rodea la muestra de mezcla asfáltica sea calentada lo suficiente para transferir calor al ligante asfáltico; mientras que la transferencia por radiación se da en el calentamiento de la superficie terrestre por el sol. Según Domínguez et al., (2019) afirman que los combustibles fósiles acarrean importantes impactos ambientales, la energía solar es absorbida por la superficie de la Tierra especialmente a nivel de pavimentos, calentándose por convección, el aire que rodea la tierra.

El probador automático de contenido de asfalto es un horno que calcina por convección forzada, en donde la mezcla es cubierta por corrientes de aire, provocando pirolisis de la muestra que se quema, pero si la temperatura disminuye, se favorece la oxidación de la mezcla. En referencia a ello, Tanimoto (2018) afirma que el envejecimiento del asfalto se produce por la incidencia de las variables ambientales o externas del medio donde se encuentra el pavimento flexible; en un corto tiempo de envejecimiento logramos determinar que en los ambientes expuestos a grandes concentraciones de sal y agua (Agua de mar e inundaciones) la estabilidad desciende al igual que el flujo, esto nos indica que las mezclas asfálticas tienden a volverse más rígidas y menos dúctiles.

\section{Ensayo para Mezclas Asfálticas envejecidas}

Afirman Vargas y Reyes (2010), que las propiedades del asfalto cambian con el tiempo, y debido a esto las especificaciones utilizadas para el diseño de las mallas viales basadas en las propiedades físicas iniciales no aseguran un buen desempeño después que el asfalto ha sido mezclado con el agregado, aplicado y puesto en marcha para soportar los esfuerzos mecánicos propios del transporte. Durante este proceso de elaboración de mezcla asfáltica, los asfaltos se 


\section{GnasisWisdam}

oxidan por acción del oxígeno y de altas temperaturas de mezclado, permitiendo que el envejecimiento inicie en forma inmediata, y posteriormente, es inducido por los diversos factores climáticos que inciden en los pavimentos.

\section{De Resistencia a la Compresión Diametral}

\section{Velocidad de aplicación de la carga en el} ensayo a tracción indirecta.

Luego de realizar las interacciones entre el tiempo de aplicación y la intensidad de carga se define la elaboración de las probetas, y se usa el método dinámico, realizando una serie de golpes sobre las caras de la probeta con la finalidad de compactar la mezcla asfáltica provocando la variación de intensidad de tránsito que se desea evaluar, recibiendo 150 golpes del martillo Marshall, requiriéndose para su elaboración entre 35 y 75 golpes.

Granados (2017) afirma que la incorporación de granos de caucho influye en la mejora significativa del comportamiento mecánico de la mezcla modificada mediante el proceso por vía seca, respecto a la mezcla asfáltica convencional, no obstante, a partir de la evaluación de los ensayos realizados, el comportamiento de la mezcla asfáltica modificada tiene los siguientes beneficios: presenta menor pérdida de resistencia por efecto del agua (Inmersión - Compresión y Tracción Indirecta), mayor resistencia a la deformación con valores de deformación permisibles, mayor cohesión y resistencia al esfuerzo cortante (Compresión Diametral), mayor resistencia al ahuellamiento y menor daño por humedad (Rueda de Hamburgo), mejora el comportamiento elástico (Módulo Resiliente), así como presenta similar comportamiento en la resistencia a la disgregación de la mezcla; los cuales se traduce en mayor durabilidad ante agentes agresores e incrementa de la vida útil del pavimento.

\section{Elaboración de probetas por el Método dinámico:}

Este método emplea la energía de compactación realizando 75 golpes en ambas caras de la probeta, de tal manera que se pueda definir las características geométricas, donde el diámetro es $101.6 \mathrm{~mm}$ y el espesor de $63.5 \mathrm{~mm}$.

Características: El contenido de material pétreo debe ser 1,100 gr. El contenido de cemento asfáltico esta expresado en porcentaje del peso del material pétreo que es $6 \%$. El contenido de cemento asfáltico es de $66 \mathrm{gr}$. La temperatura para la mezcla de cemento asfáltico (CA-60/80) debe ser de $135^{\circ} \mathrm{C}$. La temperatura de la mezcla del cemento asfáltico (Cariphalte MD 60-80) debe ser de $165^{\circ} \mathrm{C}$ La temperatura de la mezcla del material pétreo debe ser de $145^{\circ} \mathrm{C}$. Los golpes para ambas caras de la probeta deben ser de 75 .

\section{Ensayo a la tracción indirecta (Método \\ Brasileño)}




\section{GnasisWisdam}

Tayebali, et al. (1992), afirman que las mezclas asfálticas al someterse al ensayo para determinar la Resistencia a la Compresión Diametral, sometido en el rango de temperaturas entre $0^{\circ} \mathrm{C}$ a $30^{\circ} \mathrm{C}$ se obtiene la caracterización del concreto asfáltico. Se desarrolló, en el Programa de Desarrollo Estratégico de Carreteras de los Estados Unidos de Norteamérica, donde la idea es aplicar una carga verticalmente a la muestra de concreto asfáltico, produciéndose un complejo estado de esfuerzos, perpendiculares a la carga.

\section{Efecto Climático en Pavimentos}

Leyva et al. (2016) afirman que un pavimento ideal debe soportar el clima en sus variaciones estacionales; sin embargo, las altas precipitaciones pluviales, los rangos altos de variación de temperatura en el medio ambiente, sumadas a malos sistemas de bombeo y escaso mantenimiento del pavimento, provocan que en las infraestructuras viales se presenten fallas $y$ deterioros acelerados. Si queremos evitar o mitigar una prematura falla en la estructura del pavimento, se debe tener en cuenta todos los factores en el diseño que afectan al pavimento, como el contenido de humedad sobre las capas granulares y la variación de la temperatura del pavimento; sin embargo, la inclusión de estos factores requiere el uso de modelos climáticos computarizados, que permitan establecer la transferencia de calor en el que se visualice la distribución temporal y espacial de temperaturas; también, tener modelos de distribución de humedad en equilibrio a nivel de la subrasante, para predecir el grado de saturación en las capas granulares; así también, los modelos que permitan predecir el grado de saturación en las capas granulares de acuerdo a la infiltración y drenaje.

\section{Efectos de la precipitación pluvial}

La precipitación pluvial provoca los efectos en el deterioro del pavimento, debido a infiltración, condiciones geográficas, condiciones de drenaje y saturación; no obstante, la lluvia y nieve modifican la cantidad de agua por infiltración en la superficie del pavimento, ya que ingresan por infiltración a la estructura del pavimento a través de grietas en la carpeta asfáltica, generalmente a través de cunetas sin pavimentar, por terrenos más altos que la carretera, la ascensión por capilaridad desde el nivel freático, ya que siempre la capacidad portante de la estructura se degrada por la presencia de agua; lo más perjudicial es que, afecta las propiedades de los materiales en la estructura del pavimento como la resistencia al esfuerzo cortante, el módulo resiliente, la susceptibilidad a la contracción y expansión, el grado de compactación, erosión acelerada, adherencia entre agregado - asfalto y el envejecimiento del asfalto,

\section{fectaciones por los cambios de temperatura}

Las variaciones de temperatura ambiental afecta las propiedades de la capa asfáltica del pavimento y consecuentemente la estabilidad de su mezcla asfáltica, las bases estabilizadas con asfalto, 


\section{GnasisWisdam}

materiales estabilizados con algún agente viscoelástico, bases estabilizadas con cemento y losas de concreto; aunque Leyva et al., (2016), afirma que no necesariamente es lineal la relación entre la temperatura del pavimento y la temperatura del aire; debido a que el perfil de temperatura del material asfáltico requiere información de las condiciones ambientales, propiedades térmicas del asfalto y espesor de las capas de la estructura.

\subsection{El ensayo FENIX}

Regalado (2015) afirma que este ensayo consiste en determinar la resistencia a la fisuración en mezclas bituminosas, a través de sus propiedades mecánicas, entre ellas cuando se presenta la secuencia deformativa y la rotura del material, mide la energía que se disipa del material durante la fisuración, una manera de medir la resistencia a la fisuración de mezclas bituminosas; sin embargo, el ensayo desarrolla en los alrededores de la fisura del asfalto, tensiones de tracción. Las probetas que se usan para este ensayo son realizadas por el método Marshall o sobre testigos realizados en obras, por lo que se aplica a mezclas densas, semidensas, gruesas y micro aglomerados.

Al respecto Bianchetto et al. (2020) confirman que el Ensayo Fénix posibilita calcular la energía disipada a lo largo del proceso y la evaluación de esta energía es una manera positiva de medir la resistencia a la fisuración de mezclas de concreto asfáltico; sin embargo, este ensayo muestra una gigantesca versatilidad, puesto que posibilita examinar el impacto de gran proporción de cambiantes y aprender el manejo de mezclas de diversa tipología.

\section{Resultados}

Según Rondón Et al., (2016) afirman que durante el exploración experimental se analizó la influencia que tiene el ligante asfáltico sobre el fenómeno de daño por humedad en mezclas asfálticas porosas, debido solo al efecto del agua; sin embargo, el cemento asfáltico es un material impermeable y de baja reacción química con agentes externos; se reporta en este investigación que, según el tipo de ligante en presencia de agua se endurece y aumenta su rigidez; no obstante, este fenómeno podría deberse, por la presencia de moléculas de oxígeno en el agua que reacciona con los cementos asfálticos; por lo que, se deben realizar estudios que puedan validar esta hipótesis. Si esto fuere así, el envejecimiento y la inmovilidad entonces no se gestarían sólo por la interacción aire - pavimento o aire-agua-asfálto, como tradicionalmente se piensa, sino que adicionalmente el oxígeno presente en el agua podría contribuir a la generación de dicho fenómeno (interacción agua-asfalto), como tradicionalmente se piensa, y en concordancia con Regalado (2019) quien aseveró que los asfaltos sumergidos que tuvieron mayor afectación fue el ahuellamiento, toda vez que la viscosidad aumenta con el tiempo y también el aumento del módulo, 


\section{GnasisWisdam}

motivo por el que no soporta cargas, es decir, se vuelve una material muy frágil.

Así mismo en cuanto al envejecimiento se asevera que es porque, el asfalto se oxida progresivamente; no obstante, los aromáticos se reducen en $-4,53 \%$ mientras que, los asfaltenos se incrementan en un 3,42\%, provocando la conversión en resinas y asfaltenos; a diferencia de lo que manifiesta Figueroa (2015), el EF utilizada en una mezcla semi-densa y afectada por la acción del agua, presenta buena resistencia a la fisuración; no obstante, el aumento de la rigidez, la disminución de su energía de fractura y su tenacidad tienen un mayor efecto en el envejecimiento de la mezcla asfáltica.

Según Ripoll et al. (2020) aseguran que no continuamente elevar la temperatura de construcción de la mezcla de asfalto implica aumentar los impactos del medio ambiente de las mezclas bituminosas; generalmente, los impactos del medio ambiente de las mezclas bituminosas en caliente, semicalientes y templadas, los resultados conseguidos ponen de manifiesto la complejidad de fundamentarse solamente en la temperatura de construcción para clasificar las mezclas bituminosas según su eficiencia energética o el tamaño de sus impactos al medio ambiente.

\section{Discusión de resultados}

De acuerdo a Ballén (2013), el envejecimiento por oxidación altera las propiedades mecánicas de las carpetas asfálticas, tales como el aumento de la rigidez y la disminución de la tenacidad que conducen a un aumento de la fragilidad y mayor potencial para la formación de grietas, lo cual hace que se afecte la vida útil del pavimento en caso de no controlar adecuadamente el fenómeno de oxidación; ello en concordancia con Leyva et al. (2016) quienes aseveran que si queremos evitar o mitigar una prematura falla en la estructura del pavimento, se debe tener en cuenta todos los factores en el diseño que afectan al pavimento, como el contenido de humedad sobre las capas granulares y la variación de la temperatura del pavimento; de igual modo, Regalado (2019) enfatiza que los asfaltos sumergidos que tuvieron mayor afectación fueron los de ahuellamiento, toda vez que la viscosidad aumenta con el tiempo y también el aumento del módulo, motivo por el que no soporta cargas, es decir, se vuelve una material muy frágil; Aunque Leyva et al., (2016), afirman que no necesariamente es lineal la relación entre la temperatura del pavimento y la temperatura del aire, guerrero. (2019) menciona que la temperatura es el elemento de más significancia en el deterioro de una mezcla asfáltica.

\section{Conclusiones}

La existencia de agua en la superficie del pavimento, provoca desplazamiento de material fino proveniente de la capa base arrastrados a través de las grietas del pavimento. La pérdida de material, aumenta la deformación y el 


\section{GnasisWisdam}

agrietamiento, lo que acelera su deterioro; sin embargo, la presencia de agua en el deterioro acelerado se puede cuantificar a través del levantamiento de datos superficiales del pavimento que permitan identificar el comportamiento y el estado de los materiales que se degradan; es decir, el envejecimiento no se debe solamente al endurecimiento físico, sino también a procesos oxidativos; por lo que, ensayos para un proceso de envejecimiento del asfalto por oxidación en la determinación de sus propiedades respecto a la resistencia y adherencia, permite modificar las propiedades físicas de los asfaltos reológicamente que sean aptos para su utilización como materia prima más resistentes a aguas pluviales en el mercado vial.

Dentro de la infraestructura de carreteras, las etapas de construcción, conservación y mantenimiento ocasionan costos económicos (CE), siendo las dos últimas las que representan el mayor CE; sin embargo, cuando se termina la construcción de una carretera, esta se encuentra expuesta a los agentes ambientales y cargas de tráfico, lo que produce su deterioro en el tiempo y muchas veces es acelerado por el efecto del clima extremo.

Los efectos y las probables soluciones al problema de oxidación del asfalto, debería ser parte del entendimiento primordial de los expertos y técnicos que hacen el diseño de las obras de infraestructura vial como carpetas asfálticas en el territorio, lo que beneficia en gran medida la calidad y el bienestar del servicio que se presta a los usuarios de esta clase de infraestructura.

\section{Referencias:}

Arellano, L. y Cáceres, C. (2018). Importancia de las propiedades mecánicas de las mezclas asfálticas en caliente a más de $3000 \mathrm{msnm}$ para el proyecto carretera desvío Imperial - Pampas. Tesis para Titulación, Facultad de Ingeniería, Universidad Peruana de Ciencias aplicadas, Lima Peru. https://n9.cl/c00p

Ballén, D. (2013) Consideraciones para la prevención y Control de la oxidación de carpetas asfálticas en pavimentos. Tesis para grado de Magister, Universidad de Los Andes, Bogotá, Colombia. https://n9.cl/ku3e5

Bianchetto, H., Pérez, F., Amorós, J., Miró, R., Martínez, A. y Botella, R. (2020). El ensayo Fénix: una metodología efectiva para evaluar la resistencia a la fisuración en mezclas asfálticas. Rumbos Tecnológicos, 1-3, 08034 Barcelona España. https://n9.cl/0iico

Figueroa, A. (2015). Investigación sobre el efecto del agua en el asfalto y su impacto en la mezcla asfáltica. Tesis de Doctorado, Pontificia Universidad Javeriana Bogotá Colombia. https://n9.cl/hyvmf

Granados, J. (2017), Comportamiento mecánico de la mezcla asfáltica em caliente modificada com caucho mediante processo por via seca respecto a la mezcla asfáltica convencional. Tesis de Maestría, Universidad Ricardo Palma, Lima - Perú. https://n9.cl/4xc4 


\section{GnasisWisdam}

Guerrero Raga Edwin Alirio (2019) Influencia de la Temperatura y el Tiempo de Mezclado en la Modificación de un cemento asfáltico. Tesis de maestría, Universidad Santo Tomás, Bogotá, Colombia. https://n9.cl/t5lly

Hirooka, A., Vargas, F., Prado, C. y Barbosa, H. (2019). Efecto de la variación del volumen diario medio y de la tasa de crecimiento de la tasa de crecimiento en el desempeño de los pavimentos flexibles, Revista científica, Universidad estadual de Londrina. Ingeniare, 27.

Leyva, F., Camacho, E. y Aguiar, J. (2016). Simulación de Variables climáticas en ensayos de daño acelerado de pavimentos a escala Natural. Revista Infraestructura Vial, 18, 20-29. https://n9.cl/0uwfg

Mena, W. (2013). Implementación del Modelo Climático de la MEPDG "AASHTO 2008" en Colombia para tres condiciones climáticas. Tesis de Maestría en Ingeniería, Universidad EAFIT, Escuela de Ingeniería, Medellín. Recuperado el 12 de marzo de 2014. http://repository.eafit.edu.c

Mendoza, J. y Marcos, O. (2017). El efecto del cambio climático en los pavimentos. México: secretaria de comunicaciones y transportes, Publicación técnica $\mathrm{N}^{\circ}$ 104. https://n9.cl/ub25y

Ortiz, J., Crisén, X., Martínez, A., Miró, M. (2020) Impactos Ambientales de mezclas bituminosas recicladas en caliente, semicalientes y templadas.
Revista Técnica de la Asociación Española de la Carretera, Madrid España https://n9.cl/ntwy

Perera, A. (2015) Determinación y análisis de los factores climáticos críticos para el diseño de pavimentos flexibles en Costa Rica. Tesis de licenciatura en Ingeniería Civil, Universidad de Costa Rica. https://n9.cl/gj43c

Ranura, L. y Rommel, V., (2019) Evaluación Marshall y SUPERPAVE de asfalto modificado con alófano. Tesis de Titulación, Universidad Central de Ecuador, Facultad de Ingeniería Química, Quito - Ecuador. https://n9.cl/n7wsd

Regalado, F. (2015). Efecto de la Acción de agua y del envejecimiento en la resistencia a la fisuración de Mezclas Bituminosas. Tesis de Maestría, UPC Barcelona Tech. Escuela Técnica Superior de Ingeniería de Caminos, Canales y Puertos, Barcelona. Obtenido de https://n9.cl/wg832

Rondon Q. Hugo; Ruge Juan, Moreno Luis (2016). Efecto del agua sobre el asfalto y su posible influencia en el daño por humedad en una mezcla asfáltica porosa. Revista Chilena de Ingeniería Vol. 24 No.4, 2016 pp. 558-569, Santiago - Chile. https://n9.cl/i308

Tanimoto. R. (2018), Envejecimiento de mezclas asfálticas, en condiciones de laboratorio, Tesis de titulación, Universidad de San Carlos de Guatemala, Guatemala, obtenido de https://n9.cl/z0dmr 


\section{GnasisWisdam}

Vacca, H., León, M., Ruiz, D. (2012). Evaluación del efecto del envejecimiento del cemento asfáltico 80100 en el horno de película delgada sobre el comportamiento estático y dinámico de mezclas asfálticas tipo MDC-2. Ingeniería y Universidad, 16 (2), 379-396.

Vargas, X. y Reyes, F. (2010) El fenómeno de envejecimiento de Asfaltos. Revista científica: Ingeniería e investigación, 30 (3), 27-44. Universidad Manuela Beltrán - Bogotá Colombia / Pontificia Universidad Javeriana. https://n9.cl/93fsx 